\title{
Security and Optimization Challenges of Green Data Centers
}

\author{
Arif Sari, Murat Akkaya \\ Department of Management Information Systems, Girne American University, Kyrenia, Cyprus \\ Email: arifsari@gau.edu.tr, muratakkaya@gau.edu.tr
}

Received 25 October 2015; accepted 14 December 2015; published 17 December 2015

Copyright (C) 2015 by authors and Scientific Research Publishing Inc.

This work is licensed under the Creative Commons Attribution International License (CC BY). http://creativecommons.org/licenses/by/4.0/

(c) (i) Open Access

\begin{abstract}
Energy consumption in data centers has grown out of proportion in regard to the state of energy that's available in the universe. Technology has improved services and its application. The need for eco-friendly energy and increase in data centers performance brought about Green Computing into the energy consumption of data centers. Information technology has grown and eaten deep into the society that almost all the sectors if not all are dependent on information technology to move on. The consumption of power has increased greatly. In this research paper the techniques for optimizing energy in data centers for Green Computing would be discussed. This study intends to expose the limitations of existing security solutions for securing data centers by taking into consideration of limitations of existing security frameworks that cannot enhance the security of data centers.
\end{abstract}

\section{Keywords}

Green Data Centers, Data Centers, Energy Efficiency, Optimization, Security

\section{Introduction}

Green computing relates power usage in a more eco-friendly technique. Data centers consume a huge amount of energy. This contributes to the increased operational costs and also releases lots of carbon footprints into the environment. The change in climate and shortage in energy makes data centers power consumption paramount. In this research paper we are going to carry out a review on the techniques for optimizing energy in data centers for Green Computing. Researchers and scientists have proposed methods or techniques on how energy optimization can be achieved. Over the years, the use of energy in data centers increased not just in usage but also in the cost. Carbon dioxide emission is increasing in ICT infrastructure usage and also contributing to global warming, which is critically not favorable for humans and the environment at large. 
Statistics shows that the rate of carbon dioxide emission is increasing at 6\% every year, and by 2020 it could be at $12 \%$ of worldwide emission. It is important that the energy consumption of ICT infrastructure is fixed now by profiling workable methods to improve existing ones. Below is a graph of ICT infrastructure and their carbon dioxide emission rate [1] [2]. The road has become a "moving network"; today vehicles are been designed to carry networks, and communicate with other vehicles via a communication link or channel. The 2009 Urban Mobility Report, issued by the Texas Transportation Institute, reveals that in 2007, the congestion caused Urban Americans to travel 4.2 billion hours more and to purchase an extra 2.8 million gallons of fuel [1] [2]. This caused a great cost of $\$ 187.2$ billion and an increase of 50\% and above in the previous decade [1] [2].

Figure 1 shows that ICT equipment emits carbon dioxide due to energy consumption. The highest consumption rate belongs to PCs and monitors which is about $40 \%$ higher than others. The consumption of energy by ICT equipment isn't the only consideration but also from the manufacturing process to the disposal. A detailed solution to efficient usage of energy from the manufacturing to the disposal needs to be fixed [2] [3].

In this research paper, green computing is discussed in details in Section 2 and Section 3 covers the description, role and fundamental framework of data centers. Section 4 explains the green data centers and differences between green data centers and basic challenges facing by green data centers are discussed in the Section 4.1. Section 5 exposes the available techniques for optimizing the energy in green data centers while discussing different cooling methods and deployment of energy efficiency servers. Section 6 discusses about security issues in green data centers and concludes the research paper.

\section{Green Computing}

The making of computers and related resources or equipment's energy-efficient and ecofriendly brings about green technology. This includes the implementation of techniques that can increase energy optimization in data centers and reduce the carbon dioxide emission into the environment and at the same time maintain the same performance metrics. According to Daniels et al., energy consumption and the emission of carbon dioxide by ICT equipment is increasing by the double every 5years [3]-[5]. Green computing reduces cost, the carbon emission and also operational benefits. According to researchers [6]-[8], International Federation of Green and Green ICT sustainability, brings the practice of environmental sustainable IT. San Murugesan stated that Green IT refers to the study of the manufacturing, using, designing, disposing of computers, data centers, servers,

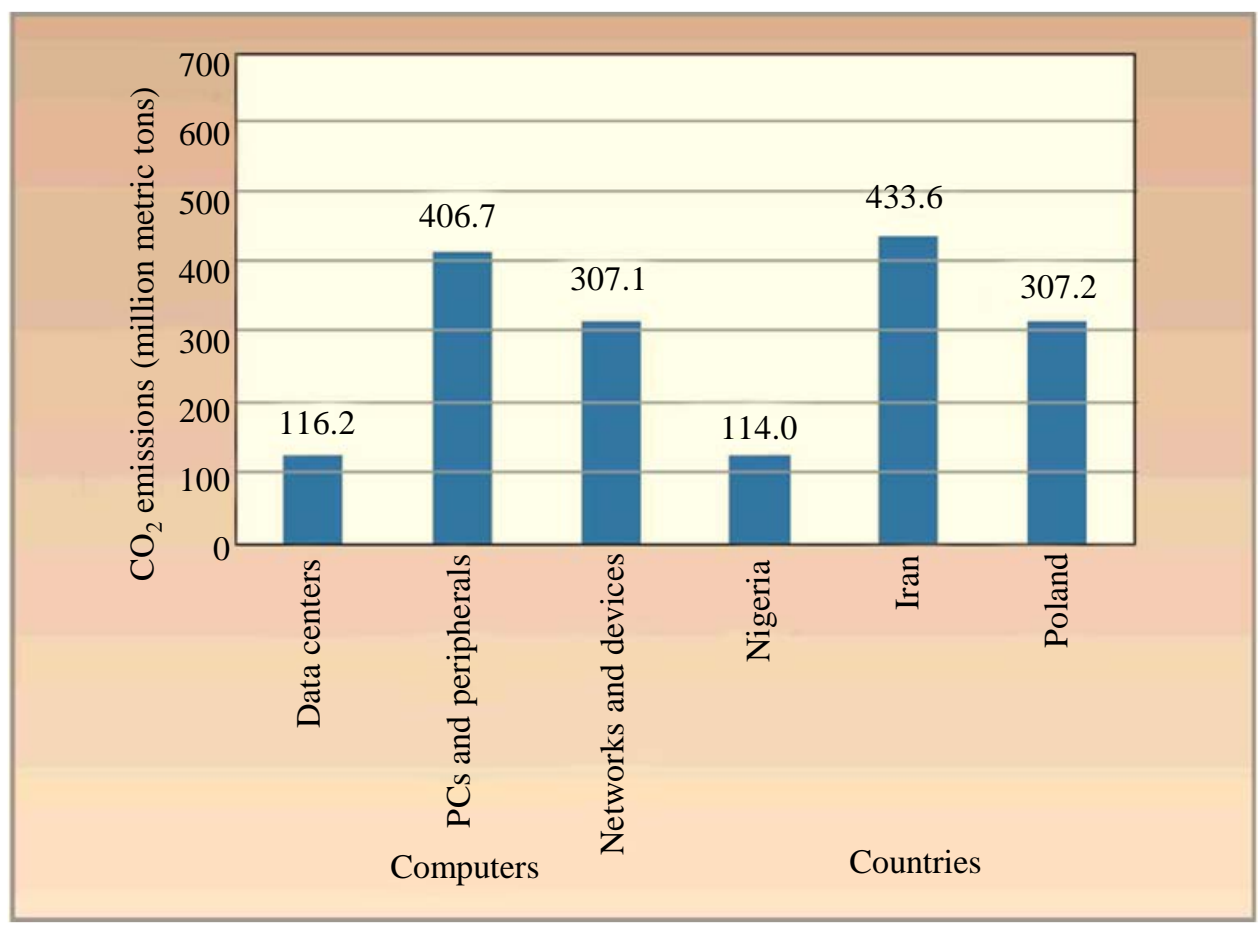

Figure 1. Computers with countries and their carbon dioxide emission. 
monitors, printers, storage device etc. in a more efficient and effective way with little or no impact on the environment [8]. It is also mentioned that, Green computing centers on the efficient use of computer and its resources [9]-[12].

The four ways on how the environment effect of computing can be introduced are [12]-[15]:

- Green use is a process of reducing the use of energy consumption by computers and other ICT systems so as to be eco-friendly.

- Green disposal refers to the refurbishing or reusing old computers.

- Green design is the design of environmental friendly computers, servers, and components.

- Green manufacturing is the producing of electronic components, computers and other subsystems. This creates minimal or no impact on the environment.

\section{Data Centers}

Data centers can be said to have started with the creation of the Electronic Numerical Integrator Computer (ENIAC). Built for the U.S Army Ballistic Research Laboratory in the year 1946, ENIAC was designed to store codes that aid in the firing of artilleries. Prior to this date, no other computer had a comparable storage and processing capacity. ENIAC had 17,468 vacuum tubes, 7,200 crystal diodes, and 10,000 capacitors. The installation of ENIAC required a floor space of 1,800 square feet and a power of $150 \mathrm{KW}$. The ENIAC computer was used until 1955 [16] [17].

As technologies began to revolutionize, better data storage machines began to emerge. Up to date, several technological breakthroughs in the creation of effective data centers have emerged, an example of this revolution is the 1.1 million square foot multi-tenant data center hub owned by Digital Realty Trust. Today, there is rapid change in the way data centers are designed. The integration of new technological solution into the operations of businesses have drastically changed designs of modern data centers. Most businesses pursue the paths of ensuring efficient levels of Information Technology service delivery yet still maintaining cost efficiency and paths to achieving business goals. For some businesses, data centers must at least ensure adequate levels of flexibility, availability and scalability. For some others, the main aim may be to achieve sufficient levels of services keeping unnecessary capital expenditures low. No matter the fundamental objective, an organization seeks to achieve with data centers, data centers have migrated to be at the center of modern software technology, serving critical roles in the expanding capabilities of organizations. Unlike traditional data centers, where the main aim was to provide a centralized data access zone which houses the large size of data, modern data center span beyond this expectation (Modern data centers have greatly improved the usability of data) [18] [19].

Data centers have enabled enterprises to achieve much more with much less, both in terms of time required to create and maintain data, and physical space. However, data centers are strategically positioned to contribute even more as technology advance with new concepts casting more light on the way data are to be used, configured and utilized. As environmental conditions continue to change, it is important that subsequent designs of data centers put into consideration this change.

These are dedicated facilities that house huge number of servers and storage systems. They serve various type or deliver different services offered to the different organizations or companies etc. There have been an increase in the number of servers in data centers (from 10,000 to 100,000 with 150,000 servers coming up), also the demand of power has spiked up from $10 \mathrm{KW} /$ rack in 2004 to $55 \mathrm{KW} /$ rack recently [20] [21]. 50\% of the power that is been used in data centers is used for cooling; this is to show how much energy requirement data centers need to perform efficiently and effectively. Arrhemius time-to-fail model, every 10 OC spike or increase in temperature in data centers increased the failure by a two factor. Data centers need to be on a regular temperature to keep the performance metrics at a stable level, and this requires lots of energy supply to the data centers.

\subsection{Role of Data Centers}

For simplicity purpose, we define Data centers as closets, rooms or sometimes entire buildings of storage for a company's processing, distribution of large amounts of data, and server information [22] [23]. No matter how small an organization is, a centralized area of data storage may be referred to as the company's data center. Data centers are usually responsible for the entire online infrastructure of a company. It is important for a company to keep and manage their information on secured servers and networks. For prompt data services, (handling the ex- 
treme amount of data traffic) data center is a key to an organization. Google handles trillions of data with high traffic. An efficient data center keeps Google up all running.

Centralization of data with the use of data centers also aids data analysis and prompt decision-making. With big data analytics, analyses of unstructured data are easier. Cloud storage ensures a robust storage of unstructured data constantly bombarding the internet. The power data centers run with helps to maintain efficient communication between transmitters and receivers [24].

\subsection{Fundamental Framework of Data Centers}

In printing out the framework of a data center, two important factors to keep in mind are efficiency and alignment with the needs of the business. There is no single "must follow" design approach in developing a data center. Different organizational requirements call for a different design approach. All data generated by the organization are stored in a secured database. With secured access, data retrieved from the database are also assumed to be secured. The whole structure showed below (Figure 2) is housed in a closet constantly powered. Depending on the design of the data center, data processing may be efficient. Data centers can be placed on a spectrum of efficiency and flexibility [25]-[27].

The data center environment is a compilation of several servers, network systems, mechanical/electrical systems, storage, applications and tools, staff and governance procedures. Depending on the requirements of the organization, data centers can go from few interconnected servers to several interconnected servers. A more advanced illustration of a data center is shown in Figure 3.

On the Figure 3, the datacenter infrastructure design is illustrated. The connection structure above illustrates the any-to-all connection structure while black lines represent the Fibre connection and blue lines are Copper. This structure runs all fibres and copper from the core horizontal distribution areas and equipment distribution areas to a central patching area. There are varieties of benefits available of this type of structured design where all server cabinets remained as locked. Since the patching is done in a central area, there is no need to enter a cabinet at any time unless there is an actual hardware change which decreases physical access to connections and leads increase in security enhancement.

In this diagram, the data center is designed to accommodate more storage space, servers and could be accessed via the internet. No matter how large (Complex) or small the data center of an organization may be, its efficiency can be measured based on the following benchmarks [28]-[30]:

1) PUE (Power Usage Effectiveness);

2) DCIE (Data Center Infrastructure Efficiency);

3) LEED Certified.

LEED is internationally recognized as a green building certification system. Providing third-party verification that a building follows stated specifications putting into consideration environmental friendly conditions: such as

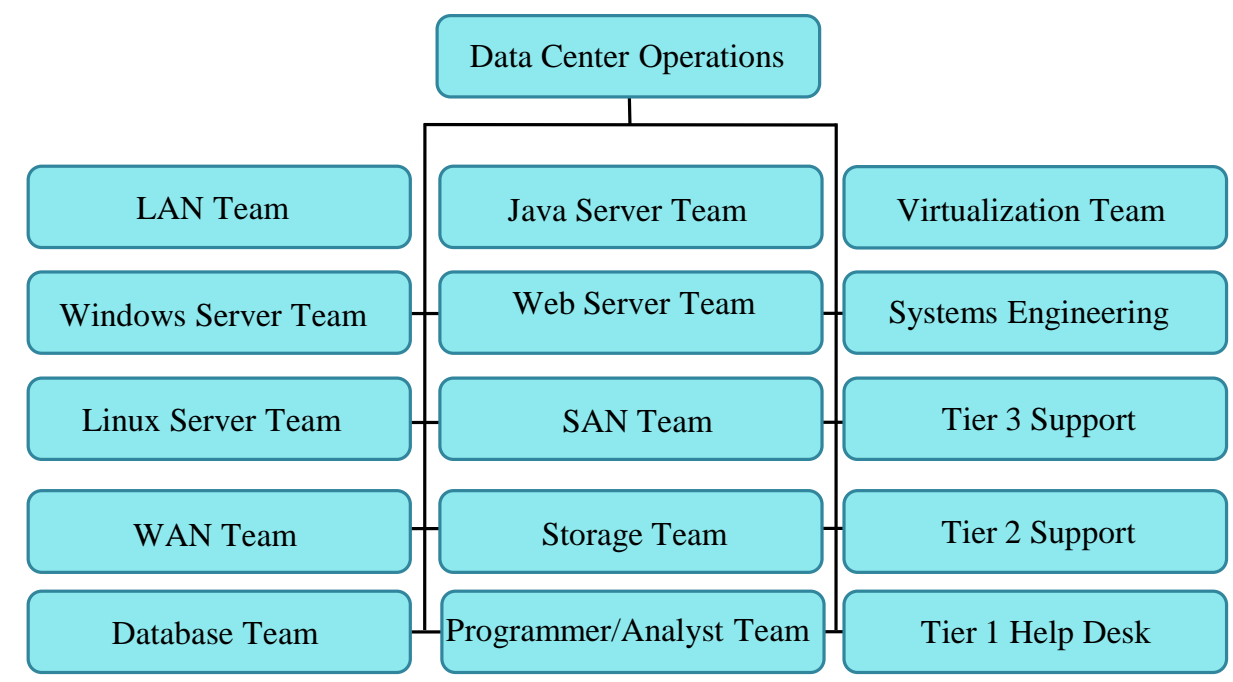

Figure 2. Structure of a data center and data center operations. 


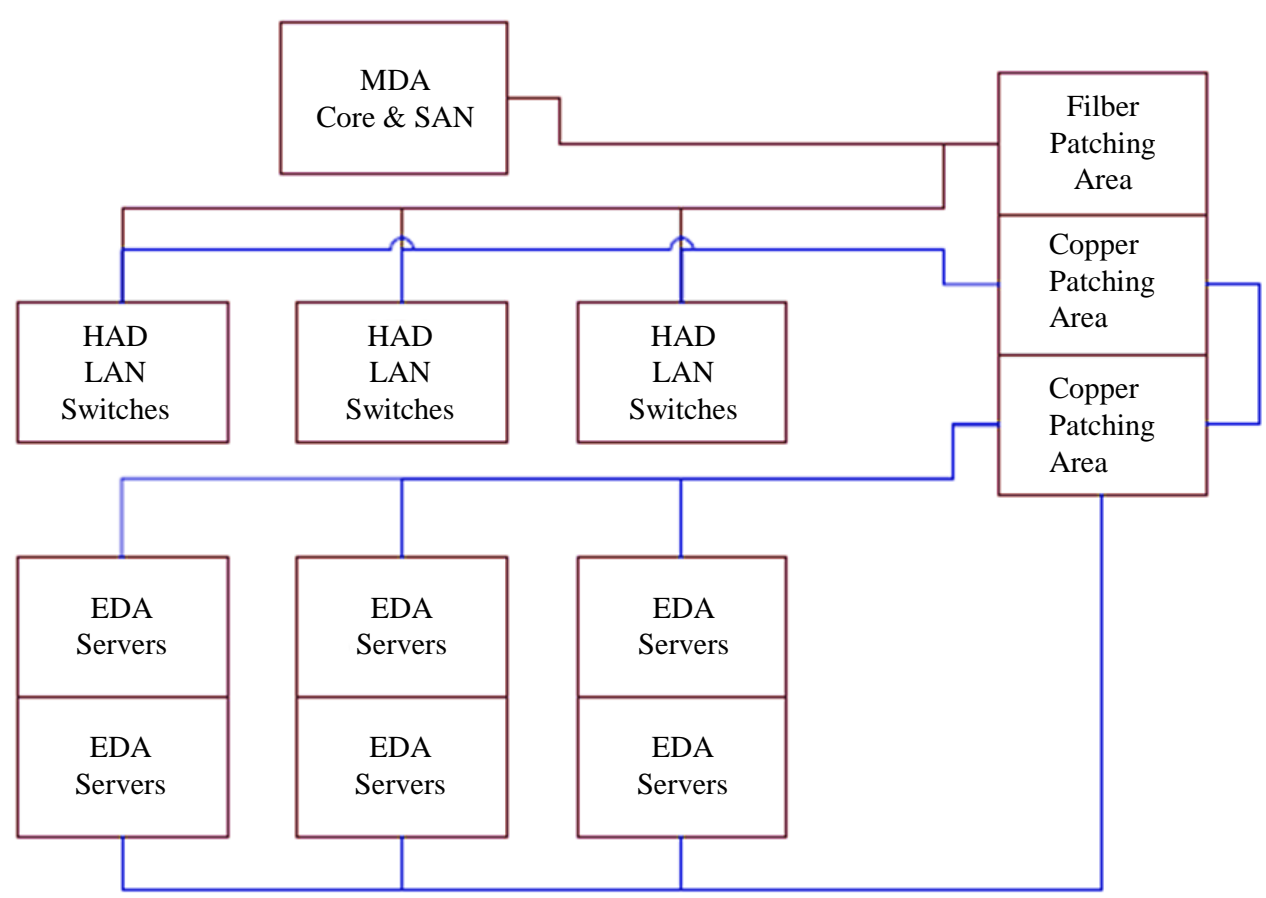

Figure 3. Datacenter infrastructure design.

energy savings, reduction of $\mathrm{CO}_{2}$ emission, the quality of the indoor environment, the stewardship of resources and their impact on the general environment.

With the aim of achieving these benchmarks, Data centers are designed considering greenhouse protection policies.

DCIE, is a standard metric used to determine the energy efficiency of a data center. DCIE's are determined to put into consideration the ratio of IT Equipment, Power: Total Facility Power compared to a $100 \%$.

\section{Green Data Centers}

A green data center operates efficiently utilizing the maximum amount of energy, causing minimum impact to the environment [31]. This takes into consideration the efficiency of all components making up the data center. The focus on green data center is driven by the need for the protection of the environment and achieving output that is more efficient by processes. Green data centers thereby are alternatives to reducing costs in comparison to non-green data centers.

\section{Challenges Facing Green Data Centers}

One of the greatest challenges of data centers is the unseen cost crisis looping within data centers, triggered by a growing divergence between prompt computation performance of the server and a lack of increase in applicable techniques and methods to rise in energy efficiency. The significant raise in server energy consumption burdens data center operators with constant increasing electricity and thus the cost of corresponding site on every host deployed (Brill, n.d). In His paper, Brill examined the economic advantage of large-scale server computing and the data centers. In his opinion, data centers create a situation where the cost of electricity and host infrastructure outpace the cost of servers. This poses a serious concern to managers as efficiency of a technology is also measured based on its consumption of electricity, known as power usage effectiveness (PUE) [32].

Another challenge faced by data centers are its carbon footprints. As man becomes more and more conscious of protecting his environment, the quantity of carbon $\left(\mathrm{CO}_{2}\right)$ released into the atmosphere is a concern to regulatory bodies. Efficiency of data centers is also measured based on its carbon usage effectiveness (CUE). With green data centers, data centers can generate power through ecofriendly methods, thereby producing $0 \%$ of $\mathrm{CO}_{2}$. For data centers that derive electricity from such sources, CUE is defined as follows [32]: 
$\mathrm{CUE}=\left(\right.$ Total $\mathrm{CO}_{2}$ emission caused by the total data center energy)/(IT Equipment Energy $)$.

Although Green data centers have reduced to great extent some of these major challenges, data centers still suffer from security challenges. Among many other challenges still facing green data centers, security is a major concern to all data managers.

\section{Techniques for Optimizing the Energy in Green Data Centers}

Data centers consumes more energy. A typical or usual datacenter consumes energy in four ways [33]:

- Critical Computational systems such as Servers, Networks, Storage etc.

- Cooling systems.

- Hosting (this includes lights and others).

- Conversion of power between Alternating and Direct current; such as the PDU (Power Distribution Units).

\subsection{Cooling Methods}

In the cooling methods the use of traditional air conditioning units can't cause a better effect or change in the temperature because of the increase in servers in data centers and more energy consumption. Even the air conditioning units also utilize energy. So a liquid cooling approach or fresh air cooling was implemented to reduce cost in the cooling system in data centers. A heat pipe based ice storage system is proposed [34]. This method of cooling system is location based, it is deployed in a geographical area where the temperature is low to enable the formation and cold water to reduce the temperature and also it reduces the consumption of energy by the data centers. The building of the ice storage system is cost effective because its location is deep underground and it requires low storage space and construction cost [34].

Warm water coming from the servers is pre-cooled using the proposed cooling model "ice storage system" it reduces the load of cooling in the chiller, this leads to a reduction in power consumption used for the data centers cooling. The transferring the waste heat to a fluid at very close the point of generation rather than transferring it to air or from point of generation to cooling system and from cooling system to air is called direct liquid cooling. The deployment of this cooling mechanism requires installation of cooling coils directly onto the rack to capture and remove waste heat. Some of the approaches proposed and mentioned about these mechanisms as it can be deployed based on water cooling system where the water circulates around the components such as processing units where the heat increase is noticeably higher.

\subsection{Energy Efficiency Servers}

Data centers require lots of energy to perform better or effectively and efficiently and this in return causes heat to spring up from the servers. One of the ways or methods is the use of renewable energy source to power up the data centers. In a geographical location where such renewable energy are sourced such as solar, wind etc. is suitable to locate a datacenter. Another method is to avoid more conversion between Alternating and Direct Current (AC/DC). A one-time conversion is ok [34].

A promising technology such as virtualization software using software known as virtual machine, that in turn provides the same input and outputs, and hardware expected kind of behavior can be used to control high server utilization of power.

In [34] and [35], various measurements metrics were proposed. This could measure and also put in quantity the performance of data centers air distribution systems. However, the downside to the proposed system is that it requires lots of tests to be carried out before it can be implemented.

There are other implementation techniques available for green computing to be realized for effective and efficient Green IT. These techniques may be applicable either requires modification for deployment to increase and enhance effectiveness and efficiency for Green IT.

Other implementation techniques are [12]:

- Virtualization.

- Scaling Unit-Based Architecture and Design Profiling the Energy Usage.

- Monitoring Systems-Wireless Sensor Networks.

- E-Waste Minimization.

- Developing thin client devices. 


\section{Security Issues in Green Data Centers}

In 21st century’s digital world, threats to data centers can come in various forms; it could come through an intruder attempting to gain physical access to the data center facility/servers, to a hack attack over a porous network [36].

Researchers have done several works and numerous solutions proposed to curtail access of unauthorized persons to data centers. However, as technology continues to advance, there is a need for the development of better security measures. Researcher's stated "Enhanced security framework to ensure data security in cloud computing using cryptography” stressed the importance to model security systems using symmetric and asymmetric cryptographic algorithms. Symmetric systems require transmitters and receivers to secretly share the same information (Keys) or one of a pair of related keys. With the use of this method of cryptography, intruders can hardly understand information transferred within a data center. However, this method of encryption possesses a major challenge among others called the key exchange problem. The key exchange problem is a problem that can arise when one party is unable to read the encrypted key. This problem can result to a classic catch-22 situation that can hinder data exchange.

Open Data Center Alliance (ODCA) in their white paper titled “Data Security Framework Rev 1.0” proposed the following security models to be followed when setting up a data center.

1) Access Control (Levels of Access Control).

2) Information classification.

3) Data Encryption and Digital Certificates: involves, Key management, Certificate management and applying confidentiality, Integrity, and available to data encryption.

4) Data Masking Techniques which involves data anonymization and tokenization Methods.

5) Security Information and event management using SIEM to Monitor Multiple Requirements.

Siemens in their white paper identified four (4) areas if given adequate attention will ensure the security of data centers, these areas include:

1) Access control.

2) Biometric access.

3) Modular system design.

4) Intelligent video surveillance.

According to their report, using an innovative technology called SIPORT these four areas are covered. The SIPORT technology promises to manage global access control, time and attendance process simply, flexibly and reliably. An extract from their white paper reads "SIPORT is a comprehensive and modular system with the highest level of reliability for time management and access control. This solution allows employees and visitors to move through a building complex without hassle while reliably keeping unauthorized persons out-in real time. We have also added a new interactive end-user interface, which allows for easy configured to suit your individual needs. This means you can use all SIPORT features easily still keeping an eye on your business at all times.

Other features SIPORT promises to offer are:

1) Networkable across the globe.

2) Open and extendable.

3) Extensive integration options.

4) Reliable and secure.

5) Modular and scalable.

The SIPORT innovation by Siemens to the security issues facing data center offers a wide solution in the sense that it addressed in detail possible attacks (Physical intruder attack and Hack attack over a network) and proffered solutions. However, most of the solutions SIPORT promises to offer are directed towards surveillance (Physical surveillance of data center facilities). This still leaves the threat of hack attacks unattended to. An expansion in the coverage of SIPORT innovation might however be a solution to unauthorized server intrusion via a network.

\section{Conclusions}

Energy optimization for data centers doesn't just deal with the efficiently use of energy but also the drop of carbon dioxide emission in the environment. The possible reduction in energy and emissions footprint through 
good design is important. The move to more environmentally sustainable applications impacts software and infrastructure architecture. The cooling system would help to control the heat and reduce the emission of footprints into the environment. Further research would be carried out as to the importance of specific techniques of energy optimization relating to data centers in green computing. A variety of methods and techniques have proposed by the researchers; however, due to high rate of complexity and lack of adoptability of these methods, energy optimization remains still a big gap for green data centers.

There is a need for in depth research to propose new cooling theories for deployment of new adoptable techniques to increase energy optimization and efficiency for Green Data Centers to support Sustainable Information Communication Technologies.

\section{References}

[1] Kumar, R. and Mieritz, L. (2007) Conceptualizing Green IT and Data Centre Power and Cooling Issues. Gartner Research Paper No. G00150322.

[2] Mishra, S. (2013) Green Computing. Science Horizon, 21.

[3] Sari, A. and Necat, B. (2012) Impact of RTS Mechanism on TORA and AODV Protocol's Performance in Mobile Ad Hoc Networks. International Journal of Science and Advanced Technology, 2, 188-191.

[4] Wang, D. (2008) Meeting Green Computing Challenges. Proceedings of 10th Electronics Packaging Technology Conference (EPTC 2008), Singapore, 9-12 December 2008, 121-126. http://dx.doi.org/10.1109/EPTC.2008.4763421

[5] Sari, A. and Necat, B. (2012) Securing Mobile Ad Hoc Networks against Jamming Attacks through Unified Security Mechanism. International Journal of Ad Hoc, Sensor \& Ubiquitous Computing, 3, 79-94. http://dx.doi.org/10.5121/ijasuc.2012.3306

[6] Daniels, G., Greene, L. and Carr, S. (2010) Planet Green, ICT for a Low-Carbon Future. Decisive Media Limited, London.

[7] Barroso, L.A. (2007) The Case for Energy-Proportional Computing. Computer, 40, 33-37. http://dx.doi.org/10.1109/MC.2007.443

[8] Murugesan, S. (2008) Harnessing Green IT: Principles and Practices. IT Professional, 10, 24-33. http://dx.doi.org/10.1109/MITP.2008.10

[9] Sari, A. (2015) Lightweight Robust Forwarding Scheme for Multi-Hop Wireless Networks. International Journal of Communications, Network and System Sciences, 8, 19-28. http://dx.doi.org/10.4236/ijcns.2015.83003

[10] Zeng, H., Ellis, C. and Lebeck, R. (2005) Experiences in Managing Energy with ECO System. IEEE Pervasive Computing, 4, 62-68. http://dx.doi.org/10.1109/MPRV.2005.10

[11] Sari, A. (2015) Security Issues in Mobile Wireless Ad Hoc Networks: A Comparative Survey of Methods and Techniques to Provide Security in Wireless Ad Hoc Networks. In: New Threats and Countermeasures in Digital Crime and Cyber Terrorism, IGI Global, Hershey, 66-94. http://dx.doi.org/10.4018/978-1-4666-8345-7.ch005

[12] Cabusao, G., Mochizuki, M., Mashiko, K., Kobayashi, T., Singh, R., Thang, N. and Xiao, W. (2010) Data Center Energy Conservation Utilizing a Heat Pipe Based Ice Storage System. Proceedings of the IEEE Symposium Components, Packaging and Manufacturing Technology, Tokyo, 24-26 August 2010, 1-4. http://dx.doi.org/10.1109/cpmtsympj.2010.5680287

[13] Sari, A. (2015) A Review of Anomaly Detection Systems in Cloud Networks and Survey of Cloud Security Measures in Cloud Storage Applications. Journal of Information Security, 6, 142-154. http://dx.doi.org/10.4236/jis.2015.62015

[14] McKenna, P. (2008) Can We Stop the Internet from Destroying Our Planet? New Scientist, January 2008

[15] Sari, A., Karaduman, A. and Firat, A. (2015) Deployment Challenges of Offshore Renewable Energy Systems for Sustainability in Developing Countries. Journal of Geographic Information System, 7, 465-477. http://dx.doi.org/10.4236/jgis.2015.75037

[16] Tozer, R. and Salim, M. (2010) Data Center Air Management Metrics-Practical Approach. Proceedings of the 12th IEEE Intersociety Conference on Thermal and Thermomechanical Phenomena in Electronic Systems (ITherm), Las Vegas, 2-5 June 2010, 1-8. http://dx.doi.org/10.1109/itherm.2010.5501366

[17] Romadhon, R., Ali, M., Mahdzir, M. and Abakr, A. (2009) Optimization of Cooling Systems in Data Centre by Computational Fluid Dynamics Model and Simulation. Proceedings of the Innovative Technologies in Intelligent Systems and Industrial Applications, Monash, 25-26 July 2009, 322-327. http://dx.doi.org/10.1109/citisia.2009.5224189

[18] Kanmdar, M.R. (2008) Literature Review: Green Computing Implementation Procedures for Energy Efficiency. http://maulik-kamdar.com/2010/08/green-computing/

[19] Brill, K.G. (n.d.) The Invisible Crisis in the Data Center: The Economic Meltdown of Moore’s Law. 
[20] Blevis, E. (2007) Sustainable Interaction Design: Invention \& Disposal, Renewal \& Reuse. In: Proceedings of the SIGCHI Conference on Human Factors in Computing Systems, ACM Press, New York, 503-512. http://dx.doi.org/10.1145/1240624.1240705

[21] Sari, A. and Onursal, O. (2013) Role of Information Security in E-Business Operations. International Journal of Information Technology and Business Management, 3, 90-93.

[22] Nathuji, R. and Schwan, K. (2007) Virtual Power: Coordinated Power Management in Virtualized Enterprise Systems. In: Proceedings of the 21st ACM SIGOPS Symposium on Operating Systems Principles, ACM Press, New York, 265278. http://dx.doi.org/10.1145/1323293.1294287

[23] Rahnama, B., Sari, A. and Makvandi, R. (2013) Countering PCIe Gen. 3 Data Transfer Rate Imperfection Using Serial Data Interconnect. Proceedings of the 2013 International Conference on Technological Advances in Electrical, Electronics and Computer Engineering (TAEECE), Konya, 9-11 May 2013, 579-582.

[24] Data Centers-Siemens (n.d.) WCMS3PortletPage. http://w3.siemens.com/topics/global/en/data-centers/pages/home.aspx

[25] Images For > Data Center Design Layout (n.d.) http://imgkid.com/data-center-design-layout.shtml

[26] Open Data Center Alliance Usage: Data Security Framework Rev 1.0 (n.d.) http://www.opendatacenteralliance.org/docs/Data_Security_Framework_Rev1.0.pdf.

[27] Sari, A. (2014) Security Issues in RFID Middleware Systems: A Case of Network Layer Attacks: Proposed EPC Implementation for Network Layer Attacks. Transactions on Networks \& Communications, Society for Science and Education, 2, 1-6.

[28] Secure Virtual Framework | hptippingpoint. (n.d.) http://www.ndm.net/ips/hp-tippingpoint/secure-virtual-framework.

[29] Sudha, M. (2012) Enhanced Security Framework to Ensure Data Security in Cloud Computing Using Cryptography. Advances in Computer Science and Its Applications, 1, 32-37.

[30] Sari, A. (2015) Two-Tier Hierarchical Cluster Based Topology in Wireless Sensor Networks for Contention Based Protocol Suite. International Journal of Communications, Network and System Sciences, 8, 29-42. http://dx.doi.org/10.4236/ijcns.2015.83004

[31] Thorsteinson, P. and Ganesh, G.G.A. (2003) Asymmetric Cryptography. http://www.informit.com/articles/article.aspx?p=102212

[32] Zeadally, A., Khan, S.A., Ullah, S. and Naveen, A.C. (2012) Energy-Efficient Networking: Past, Present, and Future. The Journal of Supercompting, 62, 1093-1118. http://dx.doi.org/10.1007/s11227-011-0632-2

[33] Sari, A., Rahnama, B. and Caglar, E. (2014) Ultra-Fast Lithium Cell Charging for Mission Critical Applications. Transactions on Machine Learning and Artificial Intelligence, 2, 11-18. http://dx.doi.org/10.14738/tmlai.25.430

[34] Obasuyi, G. and Sari, A. (2015) Security Challenges of Virtualization Hypervisors in Virtualized Hardware Environment. International Journal of Communications, Network and System Sciences, 8, 260-273. http://dx.doi.org/10.4236/ijcns.2015.87026

[35] Sari, A. and Rahnama, B. (2013) Addressing Security Challenges in WiMAX Environment. In: Proceedings of the 6th International Conference on Security of Information and Networks (SIN'13), ACM Press, New York, 454-456. http://dx.doi.org/10.1145/2523514.2523586

[36] Sari, A. and Rahnama, B. (2013) Simulation of 802.11 Physical Layer Attacks in MANET. Proceedings of the Fifth International Conference on Computational Intelligence, Communication Systems and Networks (CICSyN), Madrid, 5-7 June 2013, 334-337. http://dx.doi.org/10.1109/cicsyn.2013.79 